\title{
From the Big Bang to the Big Rip: One Cycle of a Closed Granular Friedmann-Planck Universe
}

\author{
Raymond Fèvre $(\mathbb{B}$ \\ Rue Chabot Charny, Dijon, France \\ Email: clr.fevre@wanadoo.fr
}

How to cite this paper: Fèvre, R. (2021) From the Big Bang to the Big Rip: One Cycle of a Closed Granular Friedmann-Planck Universe. Journal of High Energy Physics, Gravitation and Cosmology, 7, 377-390. https://doi.org/10.4236/jhepgc.2021.72021

Received: December 14, 2020

Accepted: March 28, 2021

Published: March 31, 202

Copyright (C) 2021 by author(s) and Scientific Research Publishing Inc. This work is licensed under the Creative Commons Attribution International License (CC BY 4.0).

http://creativecommons.org/licenses/by/4.0/ (c) (i) Open Access

\begin{abstract}
The first part of this article develops [1] a closed universe model deploying by identical multiplication a Friedmann-Planck micro-universe; thus this one constitutes the grains of the vacuum of this universe. The quantum initial expansion of this is quadratic as a function of time. Using this model, calculating the density of matter at the present time gives a correct numerical result. The essential point is that during periods of expansion following the initial quadratic period, this model reveals a surprising phenomenon. The function expressing the radius curvature as a function of time depends on the individual mass of the heaviest elementary particles created at the end of the quadratic period. The model also leads to reflection on the dark matter. The second part imagines a new type of Big Rip based on the following hypothesis: when the acceleration of the Universe, caused by dark energy, reaches the value of Planck acceleration, destruction of the microscopic structure of the Universe occurs and is replaced by a macroscopic structure (photon spheres) identical to that of the initial Planck element. Thus a new Big Bang could begin on an immensely larger scale. This reasoning eventually leads to reflection on the origins of the Big Bang.
\end{abstract}

\section{Keywords}

Big Bang, Big Rip, Friedmann Universe, Planck Scale, Dark Energy, Dark Matter, Quadratic Expansion, Cosmology, Quantum Gravity

\section{Introduction}

Most cosmological measurements indicate that the curvature of the universe is very small. Many authors conclude that the universe is flat, that is to say with zero curvature, and therefore open. That conclusion is unreasonable, because a closed universe with a very large radius of curvature compared to the Hubble ra- 
dius arrives at the same measurements. In addition, a recent article [2] suggests that our universe could have a positive curvature.

This article is based on the assumption that the vacuum of our universe is closed, granular and is originally constituted from a single element with the dimension of Planck. This idea was advanced and developed in a recent article [1], according to which the initial element would be a quantum Friedmann-Planck micro-universe, obtained from Friedmann's evolution equation transformed using conventional quantification methods into a Wheeler-de-Witt equation. In this model, the expansion of the universe corresponds to the identical multiplication of this initial element, thus constituting the quantum granular structure of the vacuum. The approach provides an explanation of the current energy of the vacuum (dark energy) and a precise calculation of its energy density. It also allows for a coherent theory of the vacuum and the elementary particles, which we know appear permanently in the vacuum as virtual particles. This approach makes it possible to calculate the elementary electric charge as well as the masses of the elementary particles.

In the first part of this article, we present a closed universe model where expansion begins with a quadratic period linked to a quantum approach to the problem. Compared to models of the universe starting with a period of inflation, this model makes it possible to define a simple physical criterion marking the end of the very rapid initial expansion and to give a correct value for the current density of matter. It also follows that subsequent evolution of the radius of curvature of the universe depends on one parameter: the individual mass of the heaviest particles created at the end of the first phase of expansion.

In the second part, the model studies the evolution of the universe, driven by dark energy, until the hypothetical appearance of a new type of Big Rip, which provides the opportunity to formulate a new hypothesis for the pre-Big Bang universe.

All reasoning is within the framework of the standard Lambda-CDM model.

\section{Closed Universe Model Starting with an Initial Quadratic Expansion}

\subsection{Model Development}

The article cited [1] assumes a primordial universe having the dimension and energy of Planck; it is therefore logical to build a model whose initial expansion occurs at constant Planck density, i.e., Planckian inflation. But such a model leads to the contradictions explained below, which affect all models of inflation. We therefore considered another hypothesis to study the initial evolution of the Universe starting from the differential equation of Friedmann, written with notations of the book [3]

$$
1+\frac{\dot{a}^{2}}{c^{2}}=\frac{8 \pi G}{3 c^{4}} \rho a^{2}
$$

An expansion of a quantum nature is considered. For this, we assume that the 
active energy (that generating the expansion) of each vacuum particle, called in [1] Friedmann-Planck micro-universe or FPmu, is defined from its Compton wavelength, which must be equal at all times to the maximum extension of the universe.

Justification of this hypothesis: the micro-universes are particles of our macro-universe. We can associate to them a Compton wavelength; this one cannot exceed the maximum extension of the Univers at any time.

Note: a designates the radius of curvature of the Universe, a function of time. All variables noted with the index p correspond to Planck units.

The quantum condition mentioned above is written:

$$
\pi a=\frac{2 \pi \hbar c}{\mathcal{E}_{v}(a)} \Rightarrow \mathcal{E}_{v}(a)=\frac{2 \hbar c}{a}
$$

FPmu is assumed to be in the form of spheres whose radius is the Planck radius. We deduce the active energy density for the expansion of the Universe.

$$
\rho_{v}(a)=\frac{3 \mathcal{E}_{v}(a)}{4 \pi a_{p}^{3}}=\frac{3 \hbar c}{2 \pi a_{p}^{3} a}
$$

Friedmann's equation is now written as:

$$
1+\frac{\dot{a}^{2}}{c^{2}}=\frac{4 a}{a_{p}}
$$

We express the solution of this differential equation, as well as the relations which follow, in two ways: first by means of the variables used and then with the decimal logarithms of these variables related to the corresponding Planck units (noted in capital letters). For the radius of curvature, when much larger than the Planck radius, we obtain:

$$
a=\frac{c^{2} t^{2}}{a_{p}}=a_{p}\left(\frac{t}{t_{p}}\right)^{2} ; \quad A=2 T
$$

The radius of curvature is therefore a quadratic function of time. A question then arises: when will this quadratic period end?

We will assume that its expiration is linked to the appearance of elementary particles from the vacuum according to the known process of creation of pairs of particles-antiparticles. The physical criterion supposed for this event is also quantum: the Compton wavelength divided by $c$ of the elementary particles that appear first must be equal to the value of the time variable reached at this moment (noted with index 1). It is therefore the elementary particles with the highest mass (the shortest wavelength) which appear first and which thus define the limit between two periods of expansion: the quadratic period and the radiative period. The stated condition results in the following relations, which fix the value of the radius of curvature reached the moment when the first elementary particles appear (we do not know if the heaviest elementary particle is ordinary or black matter): 


$$
l_{m}=\frac{2 \pi \hbar}{m c} ; l_{m}=c t_{1} ; \quad L_{m}=T_{1} ; A_{1}=2 T_{1}=2 L_{m}
$$

So we have the relationships:

$$
\frac{a_{1}}{a_{p}}=\left(\frac{t_{1}}{t_{p}}\right)^{2}=\left(\frac{l_{m}}{a_{p}}\right)^{2} ; \quad A_{1}=2 T_{1}=2 L_{m}
$$

Numerically, if the heaviest particle appearing is the d-quark, we obtain:

$$
T_{1}=L_{m}=22 ; t_{1}=5.4 \times 10^{-22} s ; A_{1}=2 T_{1}=44 ; a_{1}=1.6 \times 10^{6} \mathrm{~km}
$$

This moment of expansion corresponds to an energy transfer from the vacuum to the mass energy of the elementary particles that appear. To calculate it, we cannot refer to the energy density defined by Equation (3) because this is a quantum energy from the vacuum to the macroscopic scale. Now, at the precise moment the elementary particles appear, we seek what fraction of the internal energy of the FPmu is transferred to matter. We will use here the results of the model cited in [1] with two approaches:

a) Internal energy of the FPmu: in this case, we obtain for the vacuum energy density:

$$
\rho_{v 1}=\frac{\hbar c}{2 \pi^{2} a_{1} a_{p}^{3}}\left(\frac{3 \pi}{4}\right)^{\frac{3}{2}}(n(n+1))^{-\frac{1}{4}}
$$

$n$ is the quantum number that characterizes the FPmu; these are considered to be normally in the ground state $n=2$, corresponding to a maximum energy density. For $n=1$, the universe wave function of the Wheeler-de-Witt equation is not appropriate and therefore the FPmu cannot exist. Considering that the bursting of the micro-universes at the state 1 is the origin of the matter, we can, however, calculate the value of their internal energy density for $n=1$. By subtracting the value of the "normal" density for $n=2$, we can consider that the difference is the energy transferred from vacuum to elementary particles. Numerically, we get the following value:

$$
\rho_{m}\left(a_{1}\right)=\rho_{v 1}(n=1)-\rho_{v 1}(n=2)=\frac{0.73 \hbar c}{2 \pi^{2} a_{1} a_{p}^{3}}
$$

The total energy of matter-radiation is then:

$$
\mathcal{E}\left(a_{1}\right)=2 \pi^{2} \rho_{m}\left(a_{1}\right) a_{1}^{3}=\frac{0.73 \hbar c}{a_{p}}\left(\frac{a_{1}}{a_{p}}\right)^{2}=0.73 \mathcal{E}_{p}\left(\frac{a_{1}}{a_{p}}\right)^{2}
$$

In log-Planck terms:

$$
E_{1}=2 A_{1}-0.14=4 T_{1}-0.14
$$

\section{b) Energy of the Friedmann photon spheres composing the FPmu.}

This approach assumes that these are transformed according to model [1] at the time $t_{1}$ into Schwarzschild photons spheres which are the elementary particles. The energy of the spheres is: 


$$
\mathcal{E}_{s}=\frac{\hbar c}{18 \pi(2 n+1) a_{p}} ; n \text { integer }
$$

Taking:

$$
(2 n+1) a_{p}=a_{1} ; \quad v_{p}=\frac{4 \pi}{3} a_{p}
$$

We obtain for the energy density of the Universe, if 8 elementary particles appeared for each FPmu:

$$
\rho_{v 1}=\frac{2}{3} \cdot \frac{\hbar c}{2 \pi^{2} a_{1} a_{p}^{3}}
$$

\subsection{Radiative and Then Matter Periods}

The first of these (radiative) periods begins with the appearance of the heaviest pairs of particles, in permanent interaction with photons because their speeds are relativistic. The quantum energy of the vacuum, previously repulsive, becomes zero. The classical attractive gravity of matter and radiation succeeds it and slows expansion. We are able to justify the disappearance of vacuum energy at this instant by its quantum nature: the appearance of matter causes the collapse of the wave functions of vacuum particles (FPmu); see note b).

The lighter pairs of material particles then appear as the space expands, and evolves as a function of time during this phase where the radiation dominates according to the relationships known below; index 2 corresponds to the end of this period, the corresponding time of which is known experimentally:

$$
a \propto t^{\frac{1}{2}} ; \quad A_{2}-A_{1}=\frac{1}{2}\left(T_{2}-T_{1}\right)
$$

with regard to the total energy of the universe during the radiative period, we express the following relationships:

$$
\mathcal{E} a=C t e ; \quad E_{2}-E_{1}=-\left(A_{2}-A_{1}\right)=-\frac{1}{2}\left(T_{2}-T_{1}\right)
$$

The final (matter) period is characterized by the dominance of the mass energy of matter, the total of which remains constant as a function of time for the entire Universe. The variation of the radius of curvature as a function of time is different, and we have the following relationships (index 3 characterizes this):

$$
a \propto t^{\frac{2}{3}} ; \quad \mathcal{E}=\text { Cte } ; \quad E_{3}=E_{2} ; \quad A_{3}-A_{2}=\frac{2}{3}\left(T_{3}-T_{2}\right)
$$

We deduce from (13) the following relationships, which concern the radius of curvature and the total energy of the Universe at the present:

$$
A_{3}=\frac{3 T_{1}}{2}-\frac{T_{2}}{6}+\frac{2 T_{3}}{3} ; \quad E_{3}=\frac{9 T_{1}}{2}-\frac{T_{2}}{2}-0.14
$$

\subsection{Current Density of Matter Calculated with the Model}

The relationship giving the inverse of mass-energy density of the matter (dark + 
ordinary) at present is:

$$
\frac{1}{\rho_{3}}=\frac{2 \pi^{2} a^{3}}{\mathcal{E}_{3}}
$$

Case a): using the previous relationships, we get the following result:

$$
-R_{3}=2 T_{3}+1.44 ; \quad \frac{\rho_{P}}{\rho_{3}}=27.5\left(\frac{t_{3}}{t_{p}}\right)^{2} ;
$$

This leads to the following numerical results, which take into account the current age of the universe (13.8 billion years), determined experimentally:

$$
\begin{gathered}
\frac{t_{3}}{t_{p}}=0.8 \times 10^{61} ; T_{3}=60.9 \Rightarrow-R_{3}=123.24 \\
\frac{\rho_{p}}{\rho_{3}}=1.76 \times 10^{123} ; \quad \frac{\rho_{3}}{\rho_{p}}=0.568 \times 10^{-123}
\end{gathered}
$$

For the case $\mathrm{b}$ ), we obtain with the same calculation:

$$
\frac{\rho_{3}}{\rho_{p}}=0.520 \times 10^{-123}
$$

These two values are provided by the model for the density of matter (baryons + dark matter) at the present time. Compare this result to the experimental value, using the following data for the Planck mass density and the mass density of all matter (32\% of the total density) from cosmological measurements; see [4]:

$$
\begin{gathered}
\rho_{p}=5.1 \times 10^{93} \mathrm{~g} / \mathrm{cm}^{3}, \rho_{3}=0.270 \times 10^{-29} \mathrm{~g} / \mathrm{cm}^{3} \\
\frac{\rho_{3}}{\rho_{P}}(\exp .)=0.530 \times 10^{-123}
\end{gathered}
$$

We see a difference of $7 \%$ between the calculated value and the measured value in the case a) and a difference of $2 \%$ in the case b).

\subsection{Current Radius of Curvature and Mass of Elementary Particles}

Let us return to relations (14) and (6) which establish a link between the individual mass of the elementary particles (the heaviest) created at the end of the quadratic period and the current radius of curvature of the Universe, via the time variable

$$
\begin{gathered}
A_{3}=\frac{3 T_{1}}{2}-\frac{T_{2}}{6}+\frac{2 T_{3}}{3} ; \quad T_{1}=L_{m} \\
\frac{a_{3}}{a_{p}}=\left(\frac{l_{m}}{a_{p}}\right)^{\frac{3}{2}}\left(\frac{t_{2}}{t_{p}}\right)^{-\frac{1}{6}}\left(\frac{t_{3}}{t_{p}}\right)^{\frac{2}{3}}
\end{gathered}
$$

To make the calculation clearer, we will show the relationship between the current radius of curvature and the current Hubble radius:

$$
\frac{a_{3}}{r_{H}}=a_{H} ; \quad H R=T_{3} ; \quad A_{H}=A_{3}-T_{3}=\frac{3 L_{m}}{2}-\frac{T_{2}}{6}-\frac{T_{3}}{3}
$$


The mass of the heaviest elementary particle is also shown:

$$
\frac{l_{m}}{a_{p}}=\frac{2 \pi m_{p}}{m} ; L_{m}=\log (2 \pi)-M
$$

Thus, by calling $k$ the ratio between the mass of the particle and that of the electron, $m_{e}$, we obtain:

$$
A_{H}=-\frac{3\left(M_{e}+K\right)}{2}-\frac{T_{2}}{6}-\frac{T_{3}}{3}+\log (2 \pi) ; \quad K=\log k
$$

Numerically

$$
T_{3}=60.9 ; \quad T_{2}=55.5 ; \quad M_{e}=-22.38 \Rightarrow A_{H}=4.83-\frac{3 K}{2}
$$

We can only calculate the current radius of the Universe according to this model if we know the mass of the heaviest elementary particle category. We do not know the mass of dark matter particles. We will therefore consider only a few possible or borderline cases.

a) The d-quark $(4.8 \mathrm{MeV})$ is heavier than dark matter elementary particles: in this case, the radius of curvature of the Universe is 2344 Hubble rays, or 32350 billion light-years. It appears flat on our scale.

b) We assume an elementary dark matter particle whose mass is 250 times that of the electron (slightly heavier than the muon), or $128 \mathrm{MeV}$. In this case, we obtain a radius of curvature worth 17 times the Hubble radius, or 236 billion light-years. The curvature should appear in cosmological measurements (cf. article ref. [2]).

c) We consider a second hypothesis with an elementary dark matter particle whose mass is 1000 times that of the electron $(511 \mathrm{MeV})$. In this case, the radius of curvature is 2.14 Hubble rays, or 29.5 billion light years; this obviously does not correspond to the observed reality.

d) The fictitious limiting case with a radius of curvature equal to the Hubble radius would correspond to a particle with a mass equal to 1660 times that of the electron, or $848 \mathrm{MeV}$.

Summary table:

\begin{tabular}{cc}
\hline Mass-energy of the heaviest elementary particle & Radius of curvature of the Universe \\
\hline $4.8 \mathrm{MeV}$ (d quark) & $2344 H R=32350 \times 10^{9} \mathrm{ly}$ \\
$128 \mathrm{MeV}$ & $17 \mathrm{HR}=236 \times 10^{9} \mathrm{ly}$ \\
$511 \mathrm{MeV}$ & $2.14 H R=29.5 \times 10^{9} \mathrm{ly}$ \\
$848 \mathrm{MeV}$ & $1 H R=13.8 \times 10^{9} \mathrm{ly}$ \\
\hline
\end{tabular}

For this model, the dark matter elementary particles must not be too heavy $(<150 \mathrm{MeV})$; if so, the curvature of our Universe should be too strong, in contradiction with cosmological data.

From ref [1] we may assume hypothetic dark neutrinos and colored dark quarks (without electric charge) whose mass is $8.17 \mathrm{MeV}$. In this case, the cur- 
rent radius of curvature of our Universe should be $1060 \mathrm{HR}$. If these dark quarks subsequently compose heavier composite particles of dark matter, such as proton or neutron composed of $\mathrm{u}$ and $\mathrm{d}$ quarks, this does not change the evolution of the Universe. In an unpublished work, we find that these dark quarks perhaps compose dark matter neutral particles of $1284 \mathrm{MeV}$ mass (1.367 times the proton mass). In this case, if the Universe contains 4 such dark-matter particles for 1 baryon, we find the measured ratio between dark matter and baryonic matter (5.47); see below.

\subsection{Notes}

\section{1) Planck scale topology}

It is not contradictory to consider on one hand, each FPmu as a sphere having the radius of Planck in the entire universe, and on the other hand as a closed micro-universe whose volume is calculated accordingly. This fact is inherent in the model cited in [2]. This model considers that most of the energy of each FPmu is confined in a Friedmann-Planck volume. This requires the introduction of an additional spatial dimension "rolled up" on the Planck scale. To illustrate this idea, we can consider the two-dimensional case of a sphere with a large radius whose surface consists of small contiguous spheres of radius $r$. The area occupied by each small sphere on the large sphere is $\pi r^{2}$, but the area of each small sphere is $4 \pi r^{2}$. Likewise, each FPmu occupies a volume of $4 \pi a_{p}^{3} / 3$ within the universe and an "internal" volume consistent with its nature of Friedmann's closed space: $2 \pi^{2} a_{p}^{3}$.

\section{2) Dark quark and dark matter}

In ref [1] we present a relationship giving the masses of the elementary particles. Extending this, we assume the existence of a dark quark without electric charge whose mass is $8.17 \mathrm{MeV}$ (let's call it "duark"); see appendix below. Otherwise, in an unpublished research, we have calculated the masses of many hadrons by studying the ultra-relativistic classical motion (circular or elliptic) of the components and adding the pre quantum Bohr rule. We obtained that these masses can be written as functions of alpha and masses of the electron, the up quark and the down quark.

Examples with: $a=1 / \alpha=137.04 ; m_{e}=0.511 \mathrm{MeV} ; m_{u}=2 \mathrm{MeV} ; m_{d}=$ 4.8 $\mathrm{MeV}$; ref [5]

$$
\begin{gathered}
m(\text { pion })=2 a m_{e}=140 \mathrm{MeV}(\exp : 139.6) \\
m(\text { delta })=\frac{9 a m_{u}}{2}=1233.3 \mathrm{MeV}(\exp : 1232) \\
m(\text { neutron })=\frac{3}{4} \cdot \frac{9 a m_{u}}{2}+3 m_{d}=939.4 \operatorname{MeV}(\exp : 939.6)
\end{gathered}
$$

The two first values correspond to a circular classical motion, the third one to an elliptic motion.

Within this approach, we can assume a neutral dark particle (let's call it "darkon") composed of 3 "duarks" ( 3 different colors) whose the mass is given by: 


$$
m(\text { darkon })=\frac{3}{4} \cdot \frac{9 a m_{\text {duark }}}{6}+3 m_{\text {duark }}=1284 \mathrm{MeV}
$$

We can write now a numerical relation:

$$
\frac{4 m(\text { darkon })}{m(\text { neutron })}=5.47=\frac{26.8(\% \text { dark matter })}{4.9(\% \text { ordinary matter })}
$$

with 4 "darkons" for 1 baryon in the Universe, we have a hypothesis for the dark matter. If a part of the initial created "duarks" decay into quarks, it is possible to justify the ratio $4 / 1$.

\subsection{Discussion and Conclusion of the First Part}

This model gives the correct numerical result for the current density of the universe. This is not enough to demonstrate that our universe is closed. But there is another aspect of the question. An initial quadratic period is an interesting alternative to an inflation period. It explains why our Universe is (almost) flat, but without the arbitrary conditions of the inflation models; no need for an ad-hoc field to start the inflation and then to stop it for no reason. The quadratic period is only justified by physics laws. This is possible only for a closed and granular Universe that allows the definition of a Compton wavelength of the vacuum particles.

Moreover, the model provides the opportunity to formulate hypotheses on dark matter particles and their mass.

Note that the problems of dark matter and dark energy can be also achieved in principle in the framework of extendend gravity; see [6].

\section{Evolution of the Universe in the Future}

\subsection{Now to the Big Rip}

We know in the current period that the density of dark energy is low, but has become dominant and determines the expansion of the Universe in the form of a "soft" inflation, identical to that generated by a cosmological constant. The article cited in [1] assumes that dark energy density results from the energy of the lightest photon sphere among all those that make up each vacuum particle, and therefore that it is constant. The numerical value proposed for this density is in accordance with cosmological measurements and is currently slightly more than twice the value of the density of matter-energy given above. The Friedmann equation is written now with this value in the future when the matter-energy density becomes negligible:

$$
\begin{gathered}
1+\frac{\dot{a}^{2}}{c^{2}}=\frac{8 \pi G \rho_{v} a^{2}}{3 c^{4}} \\
\frac{\rho_{v}}{\rho_{p}}=1.15 \times 10^{-123}=\frac{3}{8 \pi \mathcal{R}} ; \text { with } \mathcal{R}=1.04 \times 10^{122} \\
1+\frac{\dot{a}^{2}}{c^{2}}=\frac{a^{2}}{\mathcal{R} a_{p}^{2}}
\end{gathered}
$$


The value of $R$ is also a theoretical result of [1], see below.

The solution of (29b) is close to the following expression, where index 0 designates the present time:

$$
a(t)=a_{0} \exp \left(\frac{t-t_{0}}{\sqrt{\mathcal{R}} t_{p}}\right)
$$

It is starting from this equation that we must consider the future of the universe. R. Caldwell proposed that an expansion of this kind [7] (but in that case a "ghost" energy of increasing density) ends in a "Big Rip", that is to say by an explosion, first of all cosmological structures, then molecules and atoms.

In the model presented here, which extends the current state of the Universe, the active density of vacuum energy remains constant. But the total energy of the Universe increases; the principle of energy conservation does not apply to vacuum. Then the acceleration of the universe increases exponentially. We will assume that when the acceleration reaches the value of the Planck acceleration, the structure of the vacuum (the FPmu) is destroyed as well as the internal structure of each vacuum particle, made up of photon spheres [1], because the inertial force of these elements exceeds the Planck force. This destruction might be immediately followed by a restructuring of the universe in the form of a Friedmann-Planck super-universe, whose dimension would be that of our universe at the time of this event. These considerations can be translated as follows:

$$
\ddot{a}\left(t_{\mathcal{R}}\right)=\frac{a_{0}}{\mathcal{R} t_{p}^{2}} \exp \left(\frac{t_{\mathcal{R}}-t_{0}}{\sqrt{\mathcal{R}} t_{p}}\right)=\frac{c}{t_{p}}
$$

where:

$$
\exp \left(\frac{t_{\mathcal{R}}-t_{0}}{\sqrt{\mathcal{R}} t_{p}}\right)=\frac{\mathcal{R} a_{p}}{a_{o}} \Rightarrow a_{\mathcal{R}}=\mathcal{R} a_{p} ; t_{\mathcal{R}} \cong \frac{\sqrt{\mathcal{R}}(\ln \mathcal{R}-C) t_{p}}{2}
$$

Relationship (32) with $C=11.8$ tells us that this type of Big Rip would take place after 135 times the current age of the Universe, or 1863 billion years.

The hypothesis for this Big Rip can be justified by returning to our Big Bang. The equations of the Friedmann-Planck micro-universe studied in the article cited in [1] are independent of the scale. They deduct the existence of photon spheres whose total number $N$ is given below (each with an energy quantized in $1 / n$ and with a near radius of the Planck radius). This result was obtained by equalizing the sum of the energies of the photon spheres to the total energy of the micro-universe:

$$
N=\exp (36 \pi \sqrt{6}-1.7)=3.75 \times 10^{119}
$$

This gives the following ratio $R$ between the energy of the micro-universe and that of the lightest photon sphere it contains, of quantum number $N$ :

$$
R=\frac{\mathcal{E}_{F}}{\mathcal{E}_{S}(N)}=36 \pi \sqrt{6} N=36 \pi \sqrt{6} \exp (36 \pi \sqrt{6}-1.7) \cong 1.04 \times 10^{122}
$$


This value allows to obtain the density of dark energy [1], it corresponds exactly to the experimental value used in (29) for the energy density of the vacuum which is actually the cause of the accelerated expansion of our Universe (equal to $3 / 8 \pi R$ of Planck energy density).

We find the same configuration, on another scale, with our Universe subjected to the Big Rip as described above. It becomes a Friedmann-Planck super-universe with a total energy given by the following relation:

$$
\mathcal{E}_{\mathcal{R}}=\sqrt{6} \mathcal{R}^{2} \mathcal{E}_{p}
$$

\subsection{The Big Rip, a New Big Bang}

In summary, our universe at the time of the Big Rip as presented here has the characteristics of a Friedmann-Planck super-universe, on a scale immensely larger than that of our starting point. The hypothesis proposed is that at that moment our universe instantly lost its microscopic structure to acquire that of a Friedmann-Planck super-universe composed of the same number $\mathrm{N}$ of photon spheres as our primordial universe, but on a different scale: that of the radius of the super-universe. This metamorphosis can be thus the beginning of a new Big Bang by identical multiplication of the FP super-universe.

This restructured super-universe has a radius of curvature, energy, and a time interval of reference given by the relations (32) and (35), which can qualify as super-units of Planck. We can immediately deduce the new "super-constants" corresponding to $G, h$ and $c$ of this primordial super-universe, which gives the following table:

$$
\begin{aligned}
& a_{\mathcal{R}}=\mathcal{R} a_{p} ; \quad t_{\mathcal{R}}=\frac{1}{2} \sqrt{\mathcal{R}}(\ln \mathcal{R}-C) t_{p} ; \quad \mathcal{E}_{\mathcal{R}}=\sqrt{6} \mathcal{R}^{2} \mathcal{E}_{p} ; \\
& c_{\mathcal{R}}=\frac{2 \sqrt{\mathcal{R}}}{\ln \mathcal{R}-C} c ; \quad \hbar_{\mathcal{R}}=\frac{\sqrt{6}}{2} \mathcal{R}^{\frac{5}{2}}(\ln \mathcal{R}-C) \hbar ; \quad G_{\mathcal{R}}=\frac{4 \sqrt{6}}{3(\ln \mathcal{R}-C)^{3}} G
\end{aligned}
$$

Can this super-universe be deployed from this initial super-element in the same way as our universe from the Planck element, and produce matter and radiation under the same conditions? The answer is affirmative if the fine structure constant alpha is independent of the scale of space, as well as the relationships between the masses of the elementary super-particles and the Planck super-mass. This is the case for the calculations presented in the article cited [1]. Alpha is the result of a scale-independent calculation of the Schwarzschild field; the same is true for the mass ratios of elementary particles to the Planck mass, which are exponential functions of alpha (see above).

The process thus described is obviously iterative: when the super-universe reaches an acceleration equal to the value "super-Planck", it metamorphoses during a new Big-Rip into the super-super-universe of Friedmann-Planck, which immediately restarts a new Big Bang. We can speak of a cyclic universe, but with a coefficient of expansion R given by the relation (34) always the same at each cycle. 


\subsection{Return to the Initial Big Bang}

If the ascending cyclical process described above is true, then it must also apply to the past of our Universe, that is, to the initial Big Bang. We can therefore imagine that the first Friedmann-Planck micro-universe at the origin of our universe results from the Big Rip of a much smaller universe having undergone its "Planck infra-acceleration". The characteristics and the fundamental constants of this infra-universe are easy to determine: it suffices to reverse the relations (36) to pass from our universe to that which preceded it. We obtain the following relationships:

$$
\begin{aligned}
& a_{-\mathcal{R}}=\frac{a_{p}}{\mathcal{R}} ; \quad t_{-\mathcal{R}}=\frac{2 t_{p}}{\sqrt{\mathcal{R}}(\ln \mathcal{R}-C)} ; \quad \mathcal{E}_{-\mathcal{R}}=\frac{\mathcal{E}_{p}}{\sqrt{6} \mathcal{R}^{2}} ; \\
& c_{-\mathcal{R}}=\frac{\ln \mathcal{R}-C}{2 \sqrt{\mathcal{R}}} c ; \quad \hbar_{-\mathcal{R}}=\frac{2 \hbar}{\sqrt{6} \mathcal{R}^{\frac{5}{2}}(\ln \mathcal{R}-C)} ; \quad G_{-\mathcal{R}}=\frac{3(\ln \mathcal{R}-C)^{3}}{4 \sqrt{6}} G
\end{aligned}
$$

Of course, for the past as for the future, we can imagine that this Planck infra-universe on the scale $1 / R$ results from a Planck infra-universe on the scale $1 / R^{2}$, according to an iterative process extended to infinity; this amounts to saying that the universe might have been born out of nothingness exclusively following the laws of Physics.

\section{Conclusions}

Let us follow our reasoning to the end. During the first Planck time of our universe, an undetermined number of infra-universes could hatch, develop and metamorphose into infra-universes on a higher scale $R$ before arriving at our universe. Our length and time scales would not in this case be arbitrary initial conditions, they would be the result of a cyclical process of increasing scale whose beginning is unknown, which can only be attributed to a chance of quantum nature.

Given the scale invariance of the processes for the appearance of matter, each of these hypothetical infra-universes of increasing size could, like our own universe, generate elementary particles, atoms, stars and planets and perhaps life and civilizations. We could call them "Gunivers" in homage to Jonathan Swift, who in the $17^{\text {th }}$ century imagined human populations existing on a different scale than ours [8].

\section{Conflicts of Interest}

The author declares no conflicts of interest regarding the publication of this paper.

\section{References}

[1] Fèvre, R. (2020) Hypotheses on Vacuum End Elementary Particles. The Friedmann-Planck Micro-Universe, Friedmann and Schwarzschild Photon Spheres. 
Journal of High Energy Physics, Gravitation and Cosmology, 6, 324-339.

[2] Di Valentino, E., Melchioerri, A. and Silk, J. (2019) Planck Evidence for a Closed Universe and a Possible Crisis for Cosmology. arXiv: 1911.02087v1 [astro-ph]

[3] Landau, L. and Lifchitz, E. (1966) Field Theory. Mir, Moscow, 416.

[4] Chardin, G. (2018) The Unsustainable Gravity of the Universe. 379.

[5] Davies, C. (2012) Standard Model Heavy Flavors Physics on the Lattice. ArXiv:1203.3862v1

[6] Corda, C. (2009) Interferometric Detection of Gravitational Waves: The Definitive Test for General Relativity. International Journal of Modern Physics D, 18, 2275-2282. https://doi.org/10.1142/S0218271809015904

[7] Caldwell, R. (2002) A Phantom Menace? Cosmological Consequences of a Dark Energy Component with Super-Negative Equation of State. Physics Letters B, 545, 23-29. https://doi.org/10.1016/S0370-2693(02)02589-3

[8] Swift, J. (1726) Gulliver's Travels. 


\section{Appendix}

Relationship giving the masses of elementary particles, before radiative corrections of Coulomb law:

$$
m \propto m_{p} \exp \left(-\frac{n a}{2 \theta}\right)
$$

The parameter $\theta$ and the quantum number $n$ characterize the elementary particle.

For the 3 elementary particles of the first family, we empirically determined the below values for $\theta$ and $n$, with the experimental mass of the electron as a reference:

Electron:

$$
\theta=3 \sqrt{2} ; \quad n=3 ; \quad m_{e}=0.511 \mathrm{MeV}
$$

Up quark:

$$
\theta=2 \sqrt{2}+3 ; \quad n=4 ; \quad m_{u}=2.13 \mathrm{MeV}
$$

Down quark:

$$
\theta=\sqrt{2}+6 ; \quad n=5 ; \quad m_{d}=4.80 \mathrm{MeV}
$$

The numerical relationship that appears between the parameters of the electric charge and the color charge of these 3 particles allows us to suppose the existence of a dark quark with parameters and mass below:

$$
\theta=3 \times 3=9 ; \quad n=6 ; \quad m_{\text {duark }}=8.17 \mathrm{MeV}
$$

